\title{
EDITORIAL:
}

\section{A UTILIZAÇÃO DO FAST-TRACK EM PUBLICAÇÕES CIENTÍFICAS}

Tempo sempre foi um fator de extrema relevância no contexto das publicações científicas. Tal relevância tem crescido ainda mais nesta segunda década do século XXI, em que os avanços científicos surgem com frequência cada vez maior, dado o aumento não só nos esforços de pesquisadores do mundo todo, mas também no reconhecimento do papel da ciência por agentes financiadores, por apoiadores e pela sociedade em geral. Neste contexto, é reforçada a atuação dos periódicos científicos como canais de divulgação das pesquisas de ponta, pois além de fazerem avançar a ciência, trazem impactos importantes para a vida das pessoas.

Apesar desse enorme peso do fator tempo, é comum pesquisadores se depararem com fluxos editoriais de periódicos que simplesmente não avançam no processo de avaliação de seus artigos. Seja por problemas internos de organização, seja pela dificuldade em manter um corpo de avaliadores ágeis e competentes, ou por quaisquer outras interferências nas rotinas internas do periódico, esse gargalo, independentemente das causas, pode trazer prejuízos: para os próprios autores, uma vez que suas pesquisas ficam "presas" ao fluxo editorial da revista escolhida, aguardando uma avaliação que pode demorar meses e até anos para chegar; para a revista, que após todo esse excesso de tempo dispendido em um processo editorial, pode vir a publicar trabalhos que não estão mais naquilo passível de ser considerado campo de ineditismo científico; e para os demais pesquisadores, que não têm acesso aos resultados das pesquisas de seus pares enquanto elas não são publicadas em canais reconhecidos.

Para tentar acelerar o fluxo editorial de revistas científicas, um caminho muitas vezes adotado por periódicos tem sido o fast-track. Trata-se de um encurtamento do tempo de avaliação dos artigos, capaz de ser estendido às demais etapas do processo editorial, reduzindo assim o tempo total desde a submissão até a publicação das pesquisas. Essa prática pode ser realizada individualmente por cada revista, que pode, por exemplo, priorizar determinados temas ou metodologias nos artigos submetidos, buscando lhes dar maior agilidade. Comumente, no entanto, essa prática se baseia em parcerias entre revistas e eventos científicos, que indicam, a partir das avaliações recebidas do corpo de avaliadores do evento, artigos que podem ser publicados por revistas, por já estarem em um processo de desenvolvimento mais avançado. Nesses casos, os periódicos se comprometem a avaliar os artigos indicados para publicação fast-track com maior velocidade, considerando que tais artigos já passaram por uma avaliação anterior, feita pelos avaliadores do próprio evento, e podem já ter incorporado as melhorias indicadas por esses avaliadores.

Diante de todos os benefícios potencialmente conseguidos com o uso do fast-track, a Contextus tem como prática editorial valorizar parcerias com este fim. Neste ano de 2019, duas delas se destacaram e têm seus artigos publicados neste número.

A primeira foi uma parceria inédita com o Encontro da Associação Nacional de PósGraduação e Pesquisa em Administração (XLIII EnANPAD), mais especificamente com a Divisão de Gestão de Operações e Logística (GOL), coordenada pela professora Dra. Susana Carla Farias Pereira (FGV/EAESP) e composto ainda de comitê científico formado pelos professores Dr. Alexandre Reis Graeml (PPGA/UTFPR), Dra. Mônica Cavalcanti Sá de Abreu (PPAC/UFC) e Dr. Ricardo Silveira Martins (CEPEAD/FACE/UFMG). Neste processo, a Contextus foi a revista escolhida para receber os melhores artigos indicados dentre os diversos 
temas que compunham essa divisão. Várias etapas de revisão e melhorias foram realizadas, incluindo uma oficina para desenvolvimento de artigos (Paper Development Workshop - PDW) durante o evento, com os professores Alexandre Graeml e Mônica Abreu, que atuaram como editores convidados da Contextus, em uma sessão que reuniu os autores para uma oficina e feedback de melhorias.

Como resultado deste fast-track, trazemos nesta edição dois artigos: um desenvolvido pelos autores Douglas Vieira Silva e Roberto Giro Moori, intitulado "PAPEL MEDIADOR DO BIG DATA ANALYTICS ENTRE GESTÃO DA CADEIA DE SUPRIMENTOS DE SERVIÇOS E DESEMPENHO OPERACIONAL NA SATISFAÇÃO DO CLIENTE”, acerca do efeito mediador do big data analytics sobre a relação entre gestão da cadeia de suprimentos de serviços e desempenho operacional, na busca pela satisfação do cliente; e o artigo desenvolvido pelos autores Gisela Gerotto, Viviane Pisano, Jacques Demajorovic, Antonio Aledo e Ana Lucia Santiago, intitulado "IMPACTO SOCIAL DA MINERAÇÃO: UMA COMPARAÇÃO ENTRE A PERCEPÇÃO DA EMPRESA E A DA COMUNIDADE", com foco na percepção de uma comunidade sobre os impactos sociais de um projeto minerário e a percepção da empresa por ele responsável.

Quanto à segunda parceria, consistiu em uma que já vem sendo mantida desde anos anteriores com o evento Seminários de Administração (XXII SemeAD), promovido pelo Programa de Pós-Graduação em Administração (PPAG) da Faculdade de Economia e Administração (FEA) da Universidade de São Paulo (USP). Nesta parceria, foram recebidas indicações de artigos para fast-track das mais diversas áreas do evento.

Como resultado deste fast-track, trazemos nesta edição três artigos: um desenvolvido pelo autor Mauro Vivaldini, intitulado "PROVEDOR DE SERVIÇOS LOGÍSTICOS PERSPECTIVAS DO MODELO 4PL", que explora e analisa a terceirização logística, com foco específico no modelo 4PL e suas perspectivas futuras; o artigo desenvolvido pelos autores Ana Luiza Leite, Dannyela da Cunha Lemos e Wilnei Aldir Schneider, intitulado "TELETRABALHO: UMA REVISÃO INTEGRATIVA DA LITERATURA INTERNACIONAL", com uma análise em bases de dados internacionais da produção científica sobre teletrabalho; e o artigo desenvolvido pelos autores Henrique Geraldo Rodrigues e Diógenes de Souza Bido, intitulado "O PROCESSO DE APRENDIZAGEM INDIVIDUAL A PARTIR DO ERRO VIVENCIADO POR GESTORES DE EMPRESAS DE BASE TECNOLÓGICA", que indica que analisar situações-problema em que se manifestam os efeitos de erros consiste em passo importante para detectar e corrigir os erros.

Desta forma, a Contextus espera reforçar ainda mais a abertura a parcerias deste tipo, atuando junto às comissões de eventos reconhecidos nas áreas de publicação da revista, tanto a nível nacional como internacional. É, portanto, um dos caminhos que pode ser buscado e, acreditamos, deve ser valorizado, por trazer benefícios a todos os envolvidos e interessados.

Por fim, este número conta ainda com quatro artigos submetidos ao processo editorial regular da Contextus, que compartilham, com a comunidade acadêmica e a sociedade em geral, relevantes achados científicos.

O primeiro, desenvolvido pelos autores Alana Pinto de Macedo da Silva e Paulo Roberto de Carvalho Nunes, intitulado "NÍVEL DE GOVERNANÇA DAS EMPRESAS ESTATAIS CONTROLADAS PELO ESTADO DO CEARÁ À LUZ DA LEI 13.303/2016 E DECRETOS ESTADUAIS REGULAMENTADORES", faz um diagnóstico do nível de governança em empresas públicas e sociedades de economia mista do Ceará, baseando-se na Lei Estadual 13.303/2016 e decretos regulamentadores. 
O segundo artigo, desenvolvido pelos autores Simone Barakat, Keysa Manuela Cunha de Mascena, Greici Sarturi e Pedro Takenouchi, intitulado "RECURSOS INTANGÍVEIS E A RELAÇÃO ENTRE DESEMPENHO SOCIAL E DESEMPENHO FINANCEIRO EM EMPRESAS BRASILEIRAS", confirma uma relação positiva entre desempenho social corporativo e desempenho financeiro corporativo, destacando recursos intangíveis como mediadores dessa relação.

O terceiro artigo, desenvolvido pelos autores Juan Arturo Castañeda-Ayarza, Cíntia Neves e André Frazão Teixeira, intitulado "PESQUISA BIBLIOGRÁFICA SOBRE OS ESTUDOS CIENTÍFICOS RELACIONADOS COM O BITCOIN E A BLOCKCHAIN", buscou mapear o conhecimento científico sobre o mercado de Bitcoin, a segurança e eficiência da Blockchain e potenciais aplicações em economia compartilhada, Big Data, Internet das Coisas e outras áreas.

O quarto artigo, desenvolvido pelos autores Chaline Evangelho Meyr, Simone Boruck Klein, Wagner Dantas de Souza Junior e Denis Dall'Asta, intitulado "EFEITO DOS COMPONENTES DO CAPITAL INTELECTUAL SOBRE O DESEMPENHO FINANCEIRO DE EMPRESAS BRASILEIRAS LISTADAS NO IBRX-50 DA B3 DE 2013 A 2017", analisou o efeito de componentes do capital intelectual, segundo o modelo VAIC ${ }^{\text {TM}}$, sobre o desempenho financeiro de empresas do índice $\mathrm{IBrX}-50$ da B3, bolsa de valores brasileira.

Ressaltamos que todo o conteúdo desta edição, bem como das anteriores, está disponibilizado de maneira gratuita, livre e irrestrita em nosso endereço eletrônico periodicos.ufc.br/contextus.

Desejamos a todos uma excelente leitura!

\section{Prof. Dr. Diego de Queiroz Machado}

Editor-chefe da Contextus (B1)

revistacontextus@gmail.com

Revista Contextus (Fortaleza) - FEAAC / UFC

Av. da Universidade, 2486 - Benfica, Fortaleza - CE - CEP 60020-180 - Brasil 
Dear readers,

We are pleased to present another issue of Contextus - Contemporary Economics and Management Journal. In this publication, articles are made available that share relevant scientific findings with the academic community and society as a whole within the areas of Administration, Accounting and Economics.

The first article, developed by the authors Alana Pinto de Macedo da Silva and Paulo Roberto de Carvalho Nunes and entitled "LEVEL OF GOVERNANCE OF STATEOWNED ENTERPRISES CONTROLLED BY CEARÁ STATE IN LIGHT OF THE LAW 13.303/2016 AND ITS STATE'S REGULATORY DECREES", diagnoses the governance level of enterprises totally or partially controlled by Ceará State, based on the State Law 13.303/2016 and its regulations.

The second article, developed by the authors Simone Barakat, Keysa Manuela Cunha de Mascena, Greici Sarturi and Pedro Takenouchi and entitled "INTANGIBLE RESOURCES AND THE RELATIONSHIP BETWEEN SOCIAL PERFORMANCE AND FINANCIAL PERFORMANCE IN BRAZILIAN COMPANIES", confirms that there is a positive relationship between corporate social performance and corporate financial performance and that intangible resources mediate this relationship.

The third article, developed by the authors Juan Arturo Castañeda-Ayarza, Cíntia Neves and André Frazão Teixeira and entitled "BIBLIOGRAPHIC RESEARCH ON SCIENTIFIC STUDIES RELATED TO BITCOIN AND BLOCKCHAIN", seeks to map the scientific knowledge about Bitcoin's market, Blockchain technology safety and efficiency, as well as potential applications in such areas as shared economy, Big Data and Internet of Things.

The fourth article, developed by the authors Chaline Evangelho Meyr, Simone Boruck Klein, Wagner Dantas de Souza Junior and Denis Dall'Asta and entitled 'EFFECT OF INTELLECTUAL CAPITAL ON THE FINANCIAL PERFORNMANCE OF BRAZILIAN COMPANIES LISTED ON B3'S IBRX-50 INDEX FROM 2013 TO 2017", analyzes the effect of intellectual capital components, in accordance with VAICTM model, on the financial performance of companies indexed by Brazilian Stock Exchange (B3) with IBrX50 .

The fifth article, developed by the authors Douglas Vieira Silva and Roberto Giro Moori and entitled "ROLE OF BIG DATA ANALYTICS FOR CUSTOMER SATISFACTION IN MEDIATING SERVICE SUPPLY CHAIN MANAGEMENT AND OPERATIONAL PERFORMANCE", examines the mediating effect of big data analytics on the relationship between service supply chain management and operational performance in the pursuit of customer satisfaction.

The sixth article, developed by the authors Gisela Gerotto, Viviane Pisano, Jacques Demajorovic, Antonio Aledo and Ana Lucia Santiago and entitled "SOCIAL IMPACT OF MINING: A COMPARISON BETWEEN THE THE COMPANY'S AND THE COMMUNITY'S PERCEPTION", compares a community's perception of a mining project's social impacts and the perception of the company responsible for the project. 
The seventh article, developed by the authors Mauro Vivaldini and entitled "LOGISTICS SERVICE PROVIDER - 4PL MODEL PERSPECTIVES", explores and analyzes logistics outsourcing, with a specific focus on the 4PL model and its future perspectives.

The eighth article, developed by the authors Ana Luiza Leite, Dannyela da Cunha Lemos and Wilnei Aldir Schneider and entitled "TELEWORKING: AN INTEGRATIVE REVIEW OF INTERNATIONAL LITERATURE", analyzes in international databases the scientific production on telework with respect to the volume and focus of study.

The ninth article, developed by the authors Henrique Geraldo Rodrigues and Diógenes de Souza Bido and entitled "THE INDIVIDUAL LEARNING PROCESS ARISING FROM ERRORS COMMITTED BY MANAGERS IN NEW TECHNOLOGY-BASED FIRMS", indicates that analyzing problem situations in which the effects of errors are manifest is an important stage for error detection and correction.

We note that the entire contents of this edition, as well as the previous ones, are free of charge and unrestrictedly available at our website periodicos.ufc.br/contextus.

We wish you an excellent read!

\section{Diego de Queiroz Machado}

Editor-in-chief of Contextus

revistacontextus@gmail.com

Contextus Journal (Fortaleza) - FEAAC / UFC

Av. da Universidade, 2486 - Benfica, Fortaleza - CE - CEP 60020-180 - Brasil 
Estimados lectores

Es con satisfacción que presentamos una edición más de Contextus - Revista Contemporánea de Economía y Administración. En esta publicación, son presentados artículos que se comparten con la comunidad académica y la sociedad en general, con resultados científicos relevantes de las áreas de administración, contabilidad y economía.

El primer artículo, desarrollado por los autores Alana Pinto de Macedo da Silva y Paulo Roberto de Carvalho Nunes, titulado "NIVEL DE GOBERNANZA DE LAS EMPRESAS ESTATALES CONTROLADAS POR EL ESTADO DEL CEARÁ A LA LUZ DE LA LEY 13.303/2016 Y SUS REGULACIONES ESTATALES", diagnostica el nivel de gobernanza de las empresas estatales controladas por el Estado de Ceará, según la Ley $13.303 / 2016$ y sus regulaciones.

El segundo artículo, desarrollado por los autores Simone Barakat, Keysa Manuela Cunha de Mascena, Greici Sarturi y Pedro Takenouchi, titulado "RECURSOS INTANGIBLES Y LA RELACIÓN ENTRE EL DESEMPEÑO SOCIAL Y EL DESEMPEÑO FINANCIERO EN EMPRESAS BRASILEÑAS", confirma no sólo que existe una relación positiva entre el desempeño social corporativo y el desempeño financiero corporativo sino también que los recursos intangibles median esta relación.

El tercer artículo, desarrollado por los autores Juan Arturo Castañeda-Ayarza, Cíntia Neves y André Frazão Teixeira, titulado "UNA PESQUISA BIBLIOGRÁFICA SOBRE CÓMO LA CIENCIA INVESTIGA EL BITCOIN Y LA TECNOLOGÍA BLOCKCHAIN", mapear el conocimiento científico desarrollado acerca del mercado de Bitcoin, la seguridad y eficiencia de la tecnología Blockchain, las potenciales aplicaciones en áreas como economía compartida, Big Data e Internet de las Cosas.

El cuarto artículo, desarrollado por los autores Chaline Evangelho Meyr, Simone Boruck Klein, Wagner Dantas de Souza Junior y Denis Dall'Asta, titulado "EFECTO DEL CAPITAL INTELLECTUAL EN EL DESEMPEÑO FINANCIERO DE LAS EMPRESAS BRASILEÑAS QUE COTIZAN EN EL IBRX-50 DE B3 DE 2013 A 2017", analiza el efecto de los componentes del capital intelectual, según el modelo VAICTM, en el desempeño financiero de las empresas del Índice IBrX-50 de B3, la bolsa de valores brasileña.

El quinto artículo, desarrollado por los autores Douglas Vieira Silva y Roberto Giro Moori, titulado "PAPEL DE MEDIACIÓN DE BIG DATA ANALYTICS ENTRE GESTIÓN DE LA CADENA DE SERVICIOS Y DESEMPEÑO OPERATIVO EN LA SATISFACCIÓN DEL CLIENTE", examina el efecto mediador del análisis de big data en la relación entre la gestión de la cadena de suministro de servicios y el rendimiento operativo en la búsqueda de la satisfacción del cliente.

El sexto artículo, desarrollado por los autores Gisela Gerotto, Viviane Pisano, Jacques Demajorovic, Antonio Aledo y Ana Lucia Santiago, titulado "IMPACTO SOCIAL DE LA MINERÍA: UNA COMPARACIÓN ENTRE LAS PERCEPCIONES DE LA EMPRESA Y DE LA COMUNIDAD", compara la percepción de una comunidad sobre los impactos sociales de un proyecto minero y la percepción de la empresa responsable del proyecto. 
El séptimo artículo, desarrollado por los autores Mauro Vivaldini, titulado "PROVEEDOR DE SERVICIOS LOGÍSTICOS - PERSPECTIVAS DEL MODELO 4PL", explora y analiza el outsourcing logístico, con un enfoque específico en el modelo 4PL y sus perspectivas futuras.

El octavo artículo, elaborado por los autores Ana Luiza Leite, Dannyela da Cunha Lemos y Wilnei Aldir Schneider, titulado "TELETRABAJO: UNA REVISIÓN INTEGRATIVA DE LA LITERATURA INTERNACIONAL", analiza la producción científica disponible en bases de datos internacionales sobre teletrabajo, para verificar el volumen de producción e identificar el foco de estudio.

El noveno artículo, elaborado por los autores Henrique Geraldo Rodrigues y Diógenes de Souza Bido, titulado EL PROCESO DE APRENDIZAJE INDVIDUAL DEL ERROR EXPERIMENTADO POR LOS GERENTES DE EMPRESAS DE BASE TECNOLÓGICA", indica que las situaciones problemáticas, donde se manifiestan los efectos de los errores, constituyen una etapa importante para detección y corrección de errores.

Destacamos que todo el contenido de esta edición, además de las anteriores, está disponible de manera gratuita, libre e irrestricta en nuestro sitio electrónico periodicos.ufc.br/contextus.

¡Deseamos a todos una gran lectura!

\section{Diego de Queiroz Machado}

Editor jefe de Contextus (B1)

revistacontextus@gmail.com

Revista Contextus (Fortaleza) - FEAAC / UFC

Av. da Universidade, 2486 - Benfica, Fortaleza - CE - CEP 60020-180 - Brasil 